\title{
LncRNA SNHGI7 Contributes to the Progression of Cervical Cancer by Targeting microRNA-375-3p
}

\author{
Shuping $\mathrm{Cao}^{1, *}$ \\ Hongxia $\mathrm{Li}^{2, *}$ \\ Lei $\mathrm{Li}^{3}$ \\ 'Department of Gynecology, Dongying \\ District People's Hospital, Dongying, \\ Shandong, People's Republic of China; \\ ${ }^{2}$ Department of Obstetrics, Dongying \\ District People's Hospital, Dongying, \\ Shandong, People's Republic of China; \\ ${ }^{3}$ Department of Pathology, Dongying \\ District People's Hospital, Dongying, \\ Shandong, People's Republic of China \\ *These authors contributed equally to \\ this work
}

Purpose: Cervical cancer is a great threat to women's health all over the world. Non-coding RNAs performed a wide range of functions. This study aimed to clarify the clinical significance and biological function of lncRNA SNHG17 and miRNA-375-3p (miR-375$3 p)$ in cervical cancer (CC).

Patients and Methods: Blood samples from $124 \mathrm{CC}$ patients and 119 healthy volunteers were collected. The relative expression of SNHG17 and miR-375-3p in CC patient serums and cells was evaluated by quantitative real-time polymerase chain reaction (qRT-PCR). The receiver operating curve (ROC) was plotted for diagnostic value estimation. The CCK-8 and transwell assay were conducted to explore the function of SNHG17 on CC cells. A luciferase reporter assay was carried out to confirm the interaction of SNHG17 and miR-375-3p. Rescue experiments were performed to verify the interaction.

Results: SNHG17 showed an ascending expression while miR-375-3p descended in the serum of CC patients. For SNHG17 and miR-375-3p, respectively, the AUC was 0.863 and 0.869 , the sensitivity was $84.7 \%$ and $75.8 \%$, and the specificity was $78.2 \%$ and $86.6 \%$. Knockdown of SNHG17 inhibited proliferation, migration, and invasion of CC cells. Serum SNHG17 expression was negatively correlated with miR-375-3p expression, and miR-375$3 p$ was the target miRNA of SNHG17. Rescue experiments verified the knockdown of SNHG17 inhibited cell growth through repressing miR-375-3p expression.

Conclusion: SNHG17 and miR-375-3p have the potential to be diagnostic markers for CC. Overexpression of SNHG17 in CC promoted the progression of CC partly via targeting miR375-3p, implying a novel therapeutic target for CC emerging.

Keywords: lncRNA SNHG17, miR-375-3p, clinical significance, biological function, cervical cancer

\section{Introduction}

Cervical cancer (CC) ranked the fourth most common cancer among women internationally, while the second most prevalent malignant neoplasm in low- and middle-income countries. ${ }^{1}$ In 2018, more than half a million new cases of cervical cancer were diagnosed and 311,365 deaths occurred worldwide due to this malignant disease. ${ }^{2,3}$ About two-thirds of the patients were diagnosed with locally advanced carcinoma of the cervix, leading to disappointing survival rates in spite of the correct comprehensive treatment. ${ }^{4}$ Coupled with the metastatic nature of cancer, cervical cancer represents poor outcomes since the moment of diagnosis. Current diagnostic tests for CC are cervical cytology test, human papillomavirus (HPV) primary screening, and contest of these two. ${ }^{5}$ But cytology test is not sensitive that caused repeat and frequent check just to prevent $\mathrm{CC}$, while the
Department of Pathology, Dongying

District People's Hospital, No. 333, Jinan

Road, Dongying, Shandong, 257000 ,

People's Republic of China

$\mathrm{Tel} / \mathrm{Fax}+86-546-8990136$

Email lilei65825@I63.com 
HPV test limited to the virus-infected $\mathrm{CC}$ and show a higher proportion of abnormal results than cytology increasing emotional distress. ${ }^{6}$ Therefore, the development of new biomolecules with specificity and sensitivity for $\mathrm{CC}$ is of vital importance to fulfill the unmet need of establishing effective diagnostic approaches in CC.

Non-coding RNAs (ncRNAs) are defined as RNA molecules that do not encode proteins. The extensive functions of ncRNA have become more and more well known, such as the control of chromosome dynamics, RNA editing, inhibition of translation. ${ }^{7}$ Usually, ncRNAs are categorized as small non-coding RNAs (primarily containing snoRNAs and miRNAs) and long non-coding RNAs (lncRNAs) based on transcript size. lncRNAs are defined as longer than 200 nucleotides non-coding RNAs and compose four-fifths of the total ncRNAs. $^{8}$ Growing evidence showed lncRNAs and miRNAs possess particular expression in different types of cancer and different pathophysiological stage of tumor growth making them a promising molecular diagnostic tool for a variety of cancers, such as lung cancer, ${ }^{9}$ breast cancer, ${ }^{10}$ colorectal cancer, ${ }^{11}$ thyroid cancer. $^{12}$ Small nucleolar RNA host gene 17 (SNHG17), one kind of 186-nt lnc RNA with chromosomal location on $20 \mathrm{q} 11.23$, has been reported with differential expressions in ovarian cancer, ${ }^{13}$ oral squamous cell carcinoma, ${ }^{14}$ lung cancer, ${ }^{15,16}$ suggesting a potential value as a biomarker in these cancers. $\mathrm{Li}$ et al have verified that SNHG17 acted as a miR-485-5p sponge to accelerate lung adenocarcinoma progression. ${ }^{15}$ In addition, SNHG17 could boost the progression of astrocytoma by binding to miR-876-5p. ${ }^{17}$ Moreover, SNHG17 was reported to regulate the initiation and progression of colon adenocarcinoma by serves as a sponge for miR-375. ${ }^{18}$ However, the expression level and potential diagnostic value of SNHG17 in $\mathrm{CC}$ have remained unexplored and amorphous.

With the aim to explore the value of SNHG17 and microRNA-375-3p (miR-375-3p), whose deregulation may facilitate pathophysiology of cervical cancer and detect a major subset of high-grade precancerous lesions of cervical scrapes at a good specificity, ${ }^{19}$ as a diagnostic marker for $\mathrm{CC}$, this study was to determine SNHG17 expression in $\mathrm{CC}$ tissues and cell lines and evaluate its diagnostic performance in $\mathrm{CC}$ patients. And its biological function in conjunction with miR-375-3p was investigated to help to understand the coherent role of SNHG17 in CC.

\section{Materials and Methods}

\section{Serum Collection from Patients and Controls}

One hundred and twenty-four CC patients who underwent histopathological examination from March 2018 to December 2020 in Dongying District People's Hospital were incorporated into the present study. Neither chemotherapy nor radiotherapy was carried out on the patients. And no other treatment history aimed at $\mathrm{CC}$ or other infectious diseases was reported by patients. The clinical stages were determined based on the International Federation of Gynecology and Obstetrics criteria (FIGO, 2009). The patient group consisted of 85 cases at stage I-II and 39 cases at III-IV. One hundred and nineteen healthy volunteers with no history of the malignant tumor were enrolled too. The protocol of this study was approved by the institutional ethics review board of Dongying District People's Hospital (no. 2,018,002). Signed written consent was acquired by the patients before the initiation of any study-related procedure. Blood samples were collected in PAXgene RNA blood collection tubes (BD, USA) from CC patients and healthy participants. All blood samples were separated by centrifugation and sera were collected, aliquoted, and immediately frozen at $-80^{\circ} \mathrm{C}$ for miRNA isolation.

\section{Cell Lines and Transfection}

The utilized four cervical cancer cell lines (SiHa, HeLa, C-33A, and MS751) and one normal cervical epithelial cells Ect1/E6E7 were all commercially obtained from Shanghai Kanglang Biological Technology Co., Ltd. All these cells were cultured in RPMI 1640 medium (Gibco by Life Technologies, USA) enriched with $10 \%(\mathrm{v} / \mathrm{v})$ fetal bovine serum (FBS) and the culture conditions were under a growth temperature $37^{\circ} \mathrm{C}$ in a $5 \% \quad \mathrm{CO}_{2}$ humidified incubator.

Small interfering negative control (si-NC (5'UUCUCCGAACGUGUCAGGU-3') and si-SNHG17 (5'GAUUGUCAGCUGACCUCUGUCCUGU-3') were purchased from Shanghai Integrated Biotech Solutions Co., Ltd (Shanghai, China). Mimic NC (5'GCGUGCUUCCGAUUGUUCUGUG-3'), miR-375-3p mimic (5'-UUUGUUCGUUCGGCUCGCGUGA-3'), inhibitor NC (5'-CAGUACUUUUGUGUAGUACAA-3'), and miR-375-3p inhibitor (miR inhibitor, 5'UCACGCGAGCCGAACGAACAAA-3') were synthesized at GenePharma (Shanghai, China). The transfection was 
performed according to Lipofectamine 3000 (Invitrogen, USA) transfection manual. Further experiments were performed after transfection for the indicated time.

\section{Quantitative Real-Time PCR (qRT-PCR)}

RNA was extracted from serum samples and cells using Trizol reagent (Invitrogen, USA), and reverse-transcribed to complementary DNA (cDNA) using PrimeScript ${ }^{\mathrm{TM}}$ RT Master Mix (Takara, Dalia, China) for SNHG17, and PrimeScript Reverse Transcriptase (Takara Bio Inc.) for miR-375-3p. The PCR assay was performed by SYBR Green qPCR Master Mix (Takara, Dalia, China) and a 7500 real-time PCR system (Applied Biosystems, USA). Primers used are supplied by GenePharma (Shanghai, China): SNHG17-fw: 5'TGCTTGTAAGGCAGGGTCTC-3'; SNHG17-rev: 5'-ACA GCCACTGAAAGCATGTG-3'; GAPDH-fw: 5'- GAAG AGAGAGACCCTCACGCTG-3'; GAPDH-rev:5'-ACTGT GAGGAGGGGAGATTCAGT-3'; miR-375-3p-fw: 5'- GTG CAGGGTCCGAGGT-3'; miR-375-3p-rev: 5'- AGCCG TTTGTTCGTTCGGCT-3'; U6-fw: 5'- CTCGCTTCGGC AGCACA-3'; U6-rev: 5'- AACGCTTCACGAATTTGCGT$3^{\prime}$. The relative expressions of SNHG17 normalized with $\mathrm{GAPDH}^{20}$ and miR-375-3p with $\mathrm{U}^{21}$ were calculated using the $2^{-\Delta \Delta \mathrm{Ct}}$ method.

\section{Cell Proliferation Assay}

The HeLa and SiHa cell lines were transfected with indicated siRNAs and collected by washing with PBS. Then, cell counting kit-8 (CCK-8; Kumamoto, Japan) was used to access the effect of SNHG17 on CC cell viability. With a density of 3000 cells/well, the cells were suspended in $100 \mu \mathrm{L}$ fresh medium and seeded into 96-well plates. After $24 \mathrm{~h}$ incubation at $37^{\circ} \mathrm{C}$ and $5 \% \mathrm{CO}_{2}(\mathrm{v} / \mathrm{v}), 10 \mu \mathrm{L} \mathrm{CCK}-8$ solution was added to the plates followed another $2 \mathrm{~h}$ incubation at $37^{\circ} \mathrm{C}$ and $5 \% \mathrm{CO}_{2}$ and the absorbance was detected at $450 \mathrm{~nm}(600 \mathrm{~nm}$ as reference wavelength) according to the microplate reader (Bio-Tek, Winooski, VT, USA) manual. The parallel test was repeated three times.

\section{Cell Migration and Invasion Assay}

Cell migration was determined using a 24-well transwell chamber (Corning, New York, USA), and cell invasion using a 24-well transwell chamber (Corning, New York, USA) coated with $50 \mathrm{mg} / \mathrm{L}$ Matrigel (BD Biosciences). Briefly, indicated-mimics transfected $\mathrm{HeLa}$ and $\mathrm{SiHa}$ cells in $100 \mu \mathrm{L}$ serum-free RPMI 1640 medium (Gibco by Life Technologies, USA) were plated in the top chamber, with a final density of $2 \times 10^{5}$ cell/well. Next,
$600 \mu \mathrm{L}$ complete medium was added to the lower chambers. Twenty-four hours later, the invaded or migrated cells were fixed with methanol, stained with crystal violet. $^{22}$ The images in five random fields were taken was by an Olympus BX53 microscope carrying DP72 camera (Olympus, Japan) and cell number was counted by Image $\mathrm{J}$ software (NIH). Parallel experiments were carried out five times independently.

\section{Apoptosis Assay}

Forty-eight-hour post-transfection, $\mathrm{SiHa}$ and Hela cells were seeded into 6-well plates at $37^{\circ} \mathrm{C}$ and $5 \% \mathrm{CO}_{2}$ After $12 \mathrm{~h}$ incubation, the cells were harvested by trypsinization without EDTA and washed with precooled PBS. Double staining was conducted by Annexin V-FITC/PI cell apoptosis kit (Solarbio, China) and propidium iodide (PI) following the protocol. Then, the cells were analyzed by MoFlo Astrios EQ flow cytometry (Beckman) for the percentage of apoptotic cells. All assay of the samples were in triplicate.

\section{Dual-Luciferase Reporter Assay}

The WT- or MUT-SNHG17 containing a miR-375-3p binding sequence was built using QuikChange SiteDirected Mutagenesis Kit (Agilent Technologies, USA) and cloned into the pmirGLO Vector (Promega, USA). Then, the construct vectors were transfected into $\mathrm{SiHa}$ cells with mimic NC, miR-375-3p mimic, inhibitor $\mathrm{NC}$, or miR-375-3p inhibitor. Subsequently, firefly and renilla (internal reference) luciferase activities were tested with a dual-luciferase reporter assay system (Promega, Madison, WI, USA).

\section{Bioinformatic Analyses of SNHG I7 Targeted miR-375-3p}

Correlation of SNHG17 with miR-375-3p was analyzed at ENCORI (http://starbase.sysu.edu.cn/).

\section{Statistical Analysis}

Statistical analyses were performed in SPSS software (SPSS Inc., Chicago, IL) and GraphPad Prism software (GraphPad Software Inc., San Diego, USA). The data were given as mean \pm standard deviation (SD). Two-tailed Student's $t$-test or one-way ANOVA with Tukey's multiple comparisons test was introduced to access the difference among two or three groups. Receiver operating characteristic (ROC) curves were used to evaluate the specificity 
and sensitivity of SNHG17 for diagnosis of CC. Pearson correlation analysis was used to measure the relationship between SNHG17 and miR-375-3p. $P$ values lower than 0.05 are considered statistically significant.

\section{Results}

\section{SNHG I7 with Elevated Expression but}

\section{miR-375-3p with Downward Expression in CC Serum and Cells}

qRT-PCR was performed to determine the relative expression of SNHG17 in CC serum and cell lines. A significantly higher expression of SNHG17 was found in the serum samples obtained from CC patients than those from subjects $(p<$ 0.01, Figure 1A). Similarly, the expressions of SNHG17 in four CC cell lines were significantly higher compared to the normal control cell (all $p<0.001$, Figure 1B). Among the four kinds of CC cells, elevated expression of SNHG17 was especially prominent in SiHa and HeLa cells. By contrast, the expression of miR-375-3p in CC samples and cells was significantly decreased when compared with the normal ones $(p<0.001$, Figure $1 \mathrm{C}$ and D).

\section{Correlation of SNHGI7 with Clinical Characteristics of CC Patients}

Based on the analysis of the Pearson's chi-squared test test, the relationship between SNHG17 expression and the clinical-pathological factors of $\mathrm{CC}$ patients was explored. The collected samples were divided into a low group $(n=60)$ and a high group $(n=64)$ based on the mean value of SNHG17 expression. From the results shown in Table 1, the expression
A

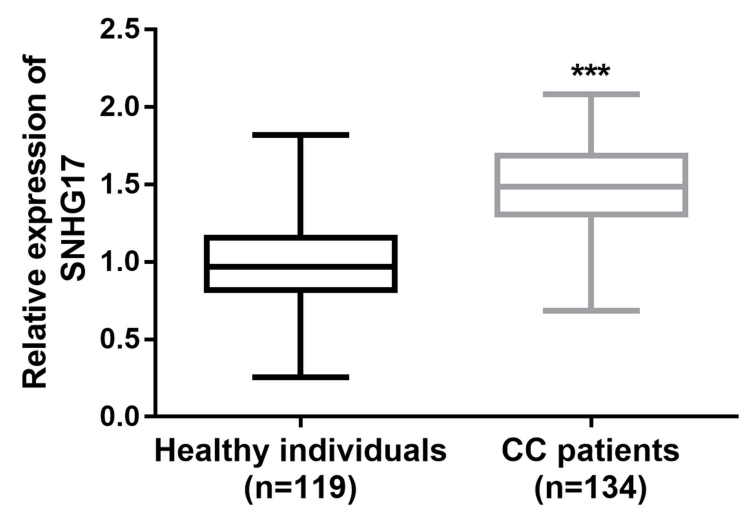

C

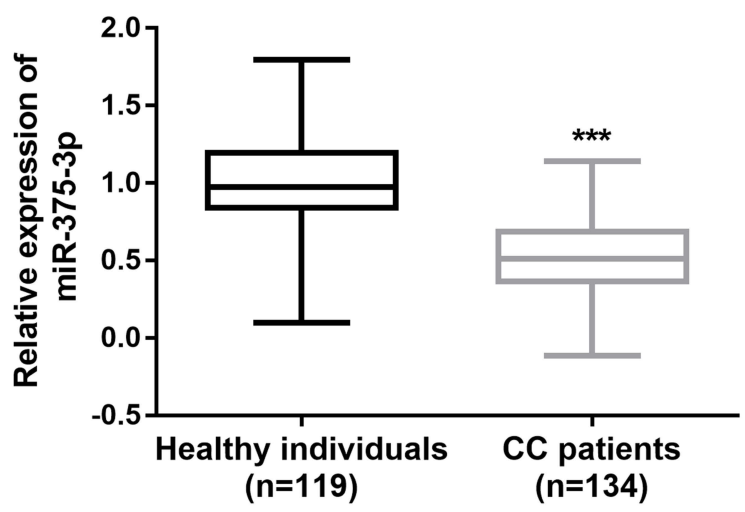

B

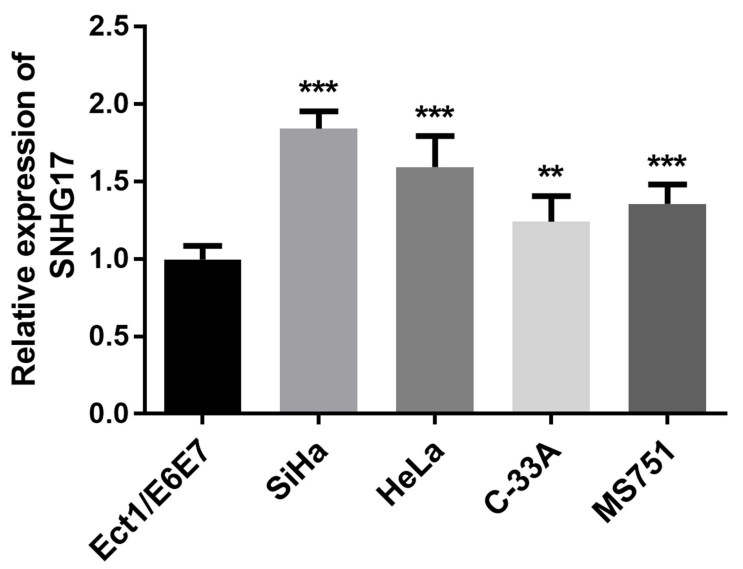

D

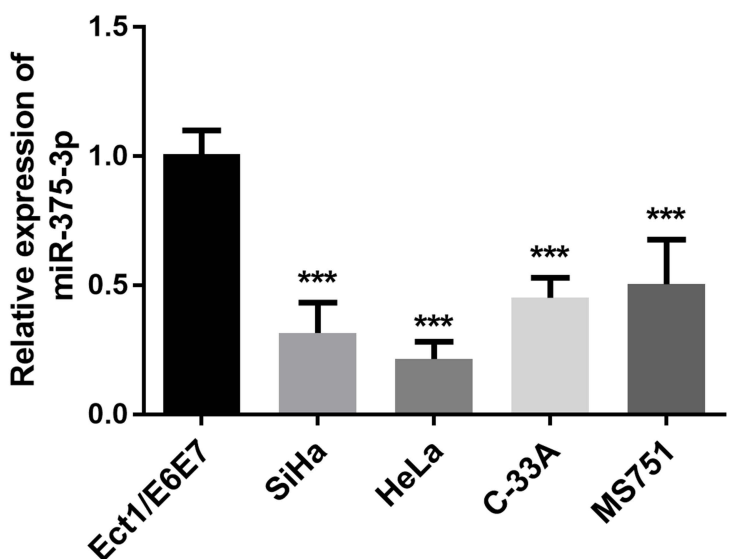

Figure I The relative expression of SNHGI7 and miR-375-3p was measured by qRT-PCR. (A) SNHGI7 expression was relatively higher in CC patient serums compared with healthy controls. (***p $<0.00 \mathrm{I}$ ). (B) SNHGI7 expression was relatively higher in CC cells compared with normal cells. (**p $<0.0 \mathrm{I}$, $* * * p<0.00 \mathrm{I}$ ). (C) miR-375-3p expression was relatively lower in CC patient serums compared with healthy controls. (***p < $0.00 \mathrm{I})$. (D) miR-375-3p expression was relatively lower in CC cells compared with normal cells. $(* * * p<0.001)$. 
Table I Correlation Between SNHGI7 Expression and Clinicopathological Characteristics in CC Patients

\begin{tabular}{|c|c|c|c|c|}
\hline \multirow[t]{2}{*}{ Parameter } & \multirow{2}{*}{$\begin{array}{l}\text { Total } \\
n=124\end{array}$} & \multicolumn{2}{|c|}{$\begin{array}{l}\text { SNHG I } 7 \text { Expression } \\
\text { Levels }\end{array}$} & \multirow[t]{2}{*}{$P$ values } \\
\hline & & $\begin{array}{l}\text { Low } \\
(n=60)\end{array}$ & $\begin{array}{l}\text { High } \\
(n=64)\end{array}$ & \\
\hline $\begin{array}{l}\text { Age } \\
\qquad \begin{array}{l}<50 \\
\geq 50\end{array}\end{array}$ & $\begin{array}{l}64 \\
60\end{array}$ & $\begin{array}{l}31 \\
29\end{array}$ & $\begin{array}{l}33 \\
31\end{array}$ & 0.991 \\
\hline $\begin{array}{l}\text { Diameter of } \\
\text { tumor } \\
\qquad<4 \mathrm{~cm} \\
\quad \geq 4 \mathrm{~cm}\end{array}$ & $\begin{array}{l}63 \\
61\end{array}$ & $\begin{array}{l}36 \\
24\end{array}$ & $\begin{array}{l}27 \\
37\end{array}$ & 0.047 \\
\hline $\begin{array}{l}\text { Differentiation } \\
\text { High-Medium } \\
\text { Low }\end{array}$ & $\begin{array}{l}70 \\
54\end{array}$ & $\begin{array}{l}34 \\
26\end{array}$ & $\begin{array}{l}36 \\
28\end{array}$ & 0.963 \\
\hline $\begin{array}{l}\text { Lymph node } \\
\text { metastasis } \\
\text { No } \\
\text { Yes }\end{array}$ & $\begin{array}{l}93 \\
31\end{array}$ & $\begin{array}{l}50 \\
10\end{array}$ & $\begin{array}{l}43 \\
21\end{array}$ & 0.038 \\
\hline $\begin{array}{l}\text { FIGO stage } \\
\text { I-II } \\
\text { III-IV }\end{array}$ & $\begin{array}{l}85 \\
39\end{array}$ & $\begin{array}{l}47 \\
13\end{array}$ & $\begin{array}{l}38 \\
26\end{array}$ & 0.023 \\
\hline $\begin{array}{l}\text { HPVI6/I8 } \\
\text { Negative } \\
\text { Positive }\end{array}$ & $\begin{array}{l}55 \\
69\end{array}$ & $\begin{array}{l}29 \\
31\end{array}$ & $\begin{array}{l}26 \\
38\end{array}$ & 0.388 \\
\hline
\end{tabular}

of SNHG17 is affected by FIGO stage ( $p=0.023)$, lymph node metastasis $(p=0.038)$ and diameter of tumor $(p=$ 0.047). However, no significant correlation was shown between the expression of SNHG17 and age, differentiation degree, or HPV16/18 infection (all $p>0.05$ ).

\section{Diagnostic Value of SNHGI7 and miR-375-3p for CC}

Further, ROC curve analyses were performed to estimate the predictive power of SNHG17 and miR-375-3p in $\mathrm{CC}$ diagnosis. As illustrated in Figure 2, both SNHG17 and miR-375-3p are effective in distinguishing $\mathrm{CC}$ patients from healthy controls. It was observed that SNHG17 had an AUC of 0.863 (sensitivity = $84.7 \%$, specificity $=78.2 \%$, and $\mathrm{miR}-375-3 \mathrm{p}$ owned an AUC of 0.869 (sensitivity $=75.8 \%$, specificity $=$ $86.6 \%$ ). These results suggest that SNHG17 and miR$375-3 p$ may be promising diagnostic biomarkers for CC.
Knock-Down of SNHGI7 Inhibits CC Cell Proliferation, Migration, and Invasion, and Induces Apoptosis

Since the abnormal expression of SNHG17 in CC serum and cells, a potential biological function of SNHG17 on the progress of $\mathrm{CC}$ would have existed. To explore this function, specifically designed siRNAs (si-NC and si- SNHG17) were transfected into $\mathrm{SiHa}$ and $\mathrm{HeLa}$ cells to downregulate SNHG17 expression $(p<0.001$, Figure $3 \mathrm{~A}$ and B). As tested using CCK-8 assays, SNHG17 inhibition obviously repressed the proliferation ability of $\mathrm{SiHa}$ and HeLa cells compared to the negative-control treatment ( $p<0.01$, Figure $3 \mathrm{C}$ and $\mathrm{D}$ ). Besides, decreased migrations were detected both in $\mathrm{SiHa}$ and HeLa cells through Transwell assay after downregulation of $\mathrm{SNHG}_{17} 7^{23}(p<0.01$, Figure 3E and F). There was a similar suppression detected in the invasion experiments $(p<0.01$, Figure $3 \mathrm{G}$ and $\mathrm{H}$ ). In the apoptosis assay by flow cytometry, the downregulation of SNHG17 dramatically increased the apoptotic cell population $(p<0.01$, Figure 3I). Based on these results, knockdown of SNHG17 significantly inhibited the proliferative, migratory, and invasive ability of $\mathrm{SiHa}$ and HeLa cells, and induced tumor apoptosis cell.

\section{miR-375-3p is a Downstream Target Gene of SNHGI7}

The predicted binding sites from ENCORI are shown in Figure 4A. ${ }^{18}$ The previous reports supported the association between SNHG17 and miR-375-3p. ${ }^{14,18}$ Expression of miR-375-3p is negatively correlated with that of SNHG17 in CC serum ( $\mathrm{r}=-0.7049, p<0.0001$; Figure 4B). Knockdown of SNHG17 could bring an increase in the expression of miR-375-3p, while si-NC not $(p<0.001$, Figure $4 \mathrm{C})$. When $\mathrm{SiHa}$ cells cotransfected with MUT-SNHG17 and miR-375-3p mimic or miR-375-3p inhibitor, there is no significant change of the relative luciferase activity compared with the cells co-transfected with MUT-SNHG17 and mimic NC and inhibitor NC. However, once the reporter vector carrying the WT-SNHG17 sequence was used, the relative luciferase activity was significantly reduced in the group co-transfected with miR-375-3p mimics and obviously increased in the group co-transfected miR$375-3 p$ inhibitor compared with a miR-NC group $(p<0.001$; Figure 4D).

In order to verify the negative correlation between SNHG17 and miR-375-3p, the function of SiHa cells was evaluated after transfected or co-transfected with si-NC, si- 

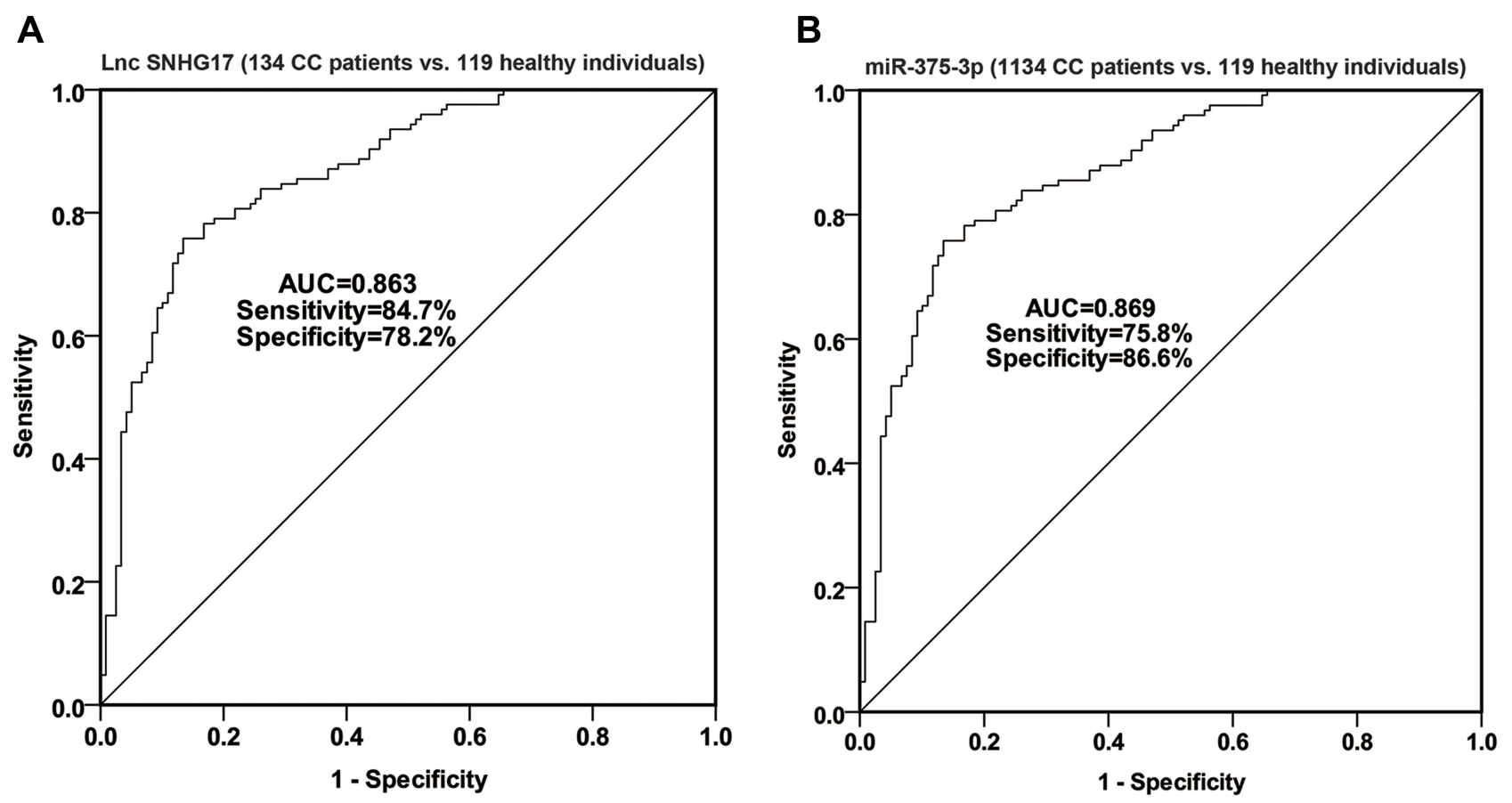

Figure 2 The ROC curve was used to analyze the diagnostic value of SNHGI7 and miR-375-3p in CC. (A) The AUC is 0.863 , sensitivity is $84.7 \%$, specificity is $78.2 \%$ for SNHGI7; (B) The AUC is 0.869 , sensitivity is $75.8 \%$, specificity is $86.6 \%$ for miR-375-3p.

SNHG17 and inhibitor NCor miR-375-3p inhibitor. Cells of the si-SNHG17 or si-SNHG17+ miR-375-3p inhibitor exhibited much higher or lower miR-375-3p relative expression than those of respective reference $(p<0.001)$, confirming that SiHa cells were all successfully transfected (Figure 5A). Optical density (OD) values from the CCK-8 assay indicated that inhibition of SNHG17 caused an obvious decrease in cell proliferation, but this decrease can be held back by a miR-375$3 p$ inhibitor $(p<0.0001$, Figure 5B). Similarly, cell migration and invasion were significantly suppressed when SNHG17 was knockdown, but the suppression could be arrested by a miR-375-3p inhibitor ( $p<0.001$, Figure $5 \mathrm{C}$ and D). In addition, miR-375-3p inhibitor can reduce the increase of apoptotic cell population caused by si-SNHG17 $(p<0.001$, Figure 5E).

\section{Discussion}

miRNA as a diagnostic marker for cancers has been studied a lot. ${ }^{24-26}$ But, in the past, lncRNAs were mostly studied about their nuclear roles. However, with the discovery of some lncRNAs with 3' cleavage and polyadenylation features, lncRNAs present the possibility of being exported to the cytoplasm since resembling the mRNA consensus sequence. These give lncRNAs cytoplasmic functions, for instance, miRNA sponging. ${ }^{27}$ This large amount of functions, together with a large number of types make lncRNAs represent many abilities, such as acting as oncogenes or tumor suppressors, even diagnostic marker in many diseases. lnc-FAM72D-3 and lnc-EPC1-4 have been identified to be potential candidate biomarkers for HCC diagnosis since it plays a novel role of hepatocarcinogenesis. $^{28}$ LncRNA lnc-GNAT1-1 was reported to inhibit gastric cancer cell proliferation and invasion in Helicobacter pylori infection. ${ }^{29}$

In this research, the expression level of SNHG17 and miR-375-3p in $\mathrm{CC}$ was studied and their value for $\mathrm{CC}$ diagnosis was accessed. SNHG17 turned out to be overexpression but miR-375-3p showed a down-regulated expression in CC patients and cells by qRT-PCR. As to ROC curves, SNHG17 and miR-375-3p represented both good sensitivity and specificity for the diagnosis of CC. With the chi-square test, it is found that SNHG17 overexpression was significantly correlated with FIGO stage, lymph node metastasis, and diameter of tumor in $\mathrm{CC}$ patients. So, our results showed SNHG17 and miR-375$3 p$ was a prospective diagnostic marker for $\mathrm{CC}$, implied that SNHG17 and can be developed to diagnostic new molecular markers in CC. Recently, SNHG17 was also 
A

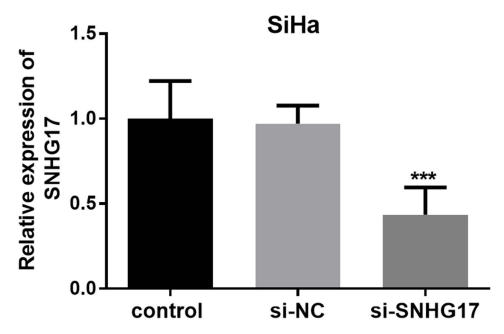

D

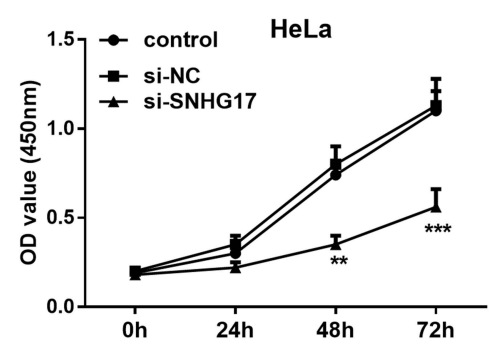

G

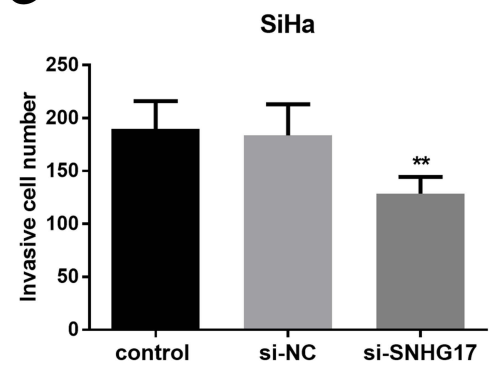

B

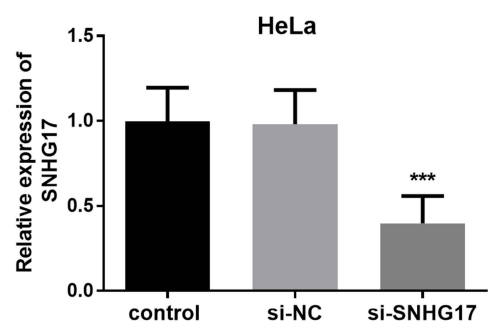

E

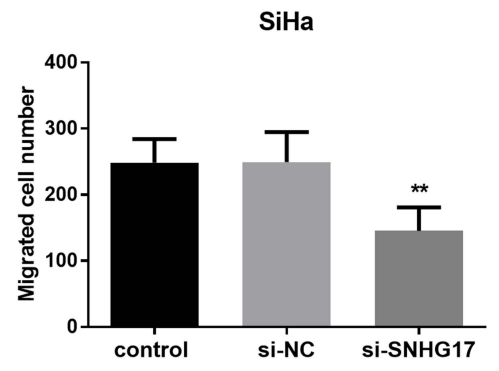

H

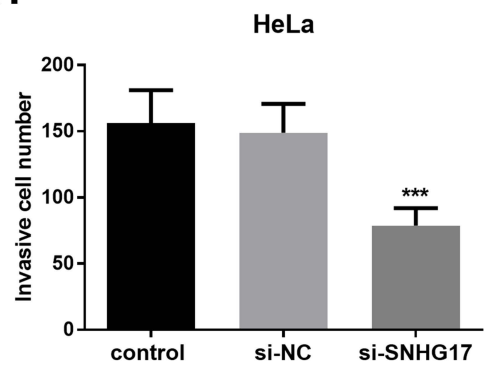

C

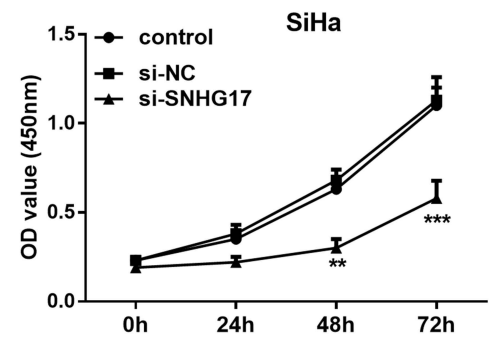

F

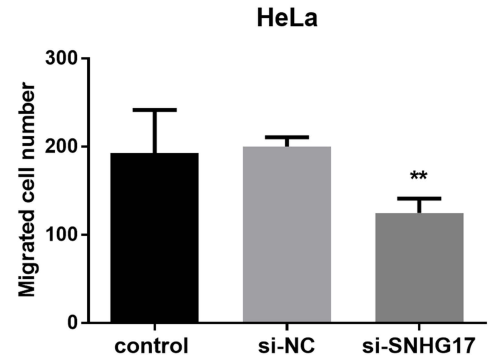

I

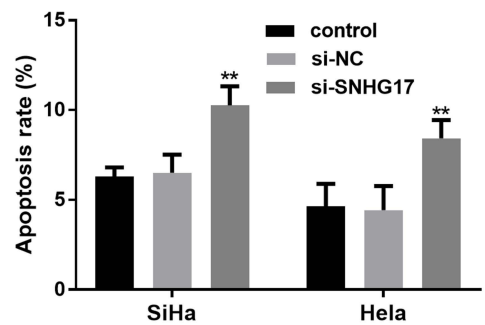

Figure 3 The effect of the SNHGI7 expression on the proliferative, migrative, and invasive capacity of CC cells. (A and B) si-SNHGI7 can inhibit the expression of SNHGI 7 in SiHa and HeLa cells (***p $<0.00 \mathrm{I}$ ). (C and D) Downregulation of SNHG I 7 restrain cell proliferation of SiHa and HeLa cells $(* * p<0.0 \mathrm{I}$, ***p $<0.00 \mathrm{I})$. (E and $\mathbf{F}$ ) Knockdown of SNHGI7 inhibit CC SiHa and HeLa cell migration $(* * p<0.0 \mathrm{I})$. (G and $\mathbf{H})$ Knockdown of SNHGI7 suppress tumor cell invasion $(* * p<0.0 \mathrm{I}$, *** $p<0.00 \mathrm{I})$. (I) Downregulation of SNHGI7 induced apoptosis of SiHa and HeLa cells $(* * p<0.01$ ).

reported with overexpression in colorectal cancer tissues and gastric cancer tissues and then can be provided as a prognostic factor for colorectal cancer and gastric cancer. $^{20,30}$

A mounting number of studies have revealed that aberrant expression levels of ncRNAs may function in cell biological behaviors. To further discover the function of SNHG17 in CC, si-SNHG17 was used to knockdown the expression of SNHG17 in SiHa and HeLa cells, and then proliferation, migration, and invasion of both two kinds of CC cells were inhibited after knockdown of SNHG17. These findings indicate that SNHG17 plays an important role in promoting the development of $\mathrm{CC}$, and may act as biological indicators for the clinical progression and diagnosis of CC. Much recent research in other cancers supported this finding. SNHG17 with high expression in colorectal adenocarcinoma can promote the proliferation and migration of colorectal adenocarcinoma cells. ${ }^{31}$ Knockdown of SNHG17 decreases prostate cancer cell proliferation, invasion, migration, and epithelialto-mesenchymal transition (EMT) but strengthened apoptosis, to promote prostate cancer progression. ${ }^{32}$ Besides, SNHG17 also promotes the progression of breast cancer, ${ }^{33}$ ovarian cancer, ${ }^{34}$ and oral squamous cell carcinoma. ${ }^{14}$ All these studies implied that miR1298 may play an important role in the progression of various cancers. Since miR-375-3p has been reported to affect the development of HPV16-positive human $\mathrm{CC}$ cells and the mechanism has been explored further, this study does not repeat. ${ }^{35}$ 


\section{A}

SNHG17: 5' ACCAUCAGUAAUGUCGAACAAA miR-375: 3' AGUGCGCUCGGCUUGCUUGUUU

\section{C}

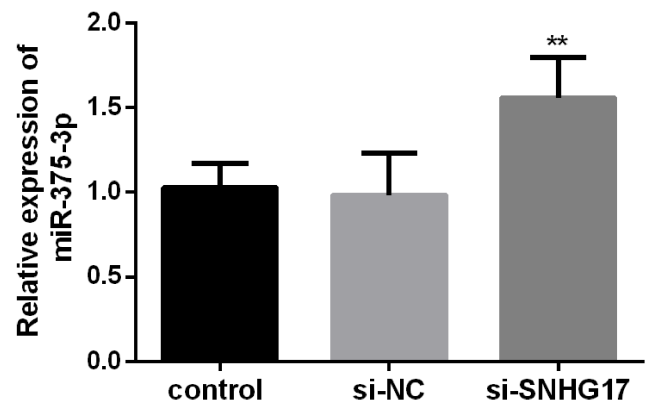

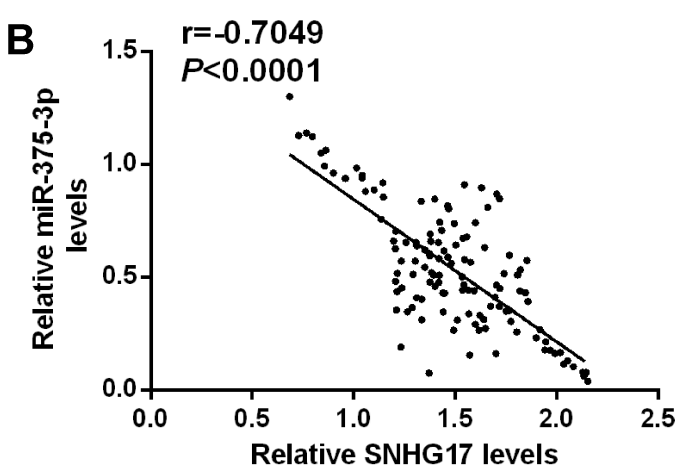

D

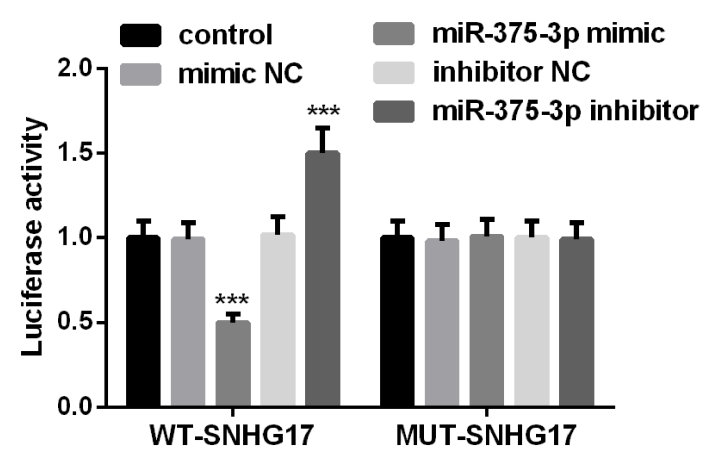

Figure 4 miR-375-3p is a target miRNA of SNHG I7. (A) Predicted binding sites of SNHGI7 and miR-375-3p. (B) The correlation between the expression of SNHGI7 and miR-375-3p is negative in CC serum. ( $r=-0.7049, p<0.000$ I) (C) Knockdown of SNHGI7 can release the expression of miR-375-3p. (D) Dual-luciferase reporter assay was performed in $\mathrm{SiHa}$ cells. $* * p<0.01$, *** $p<0.001$.

As is known to us, one reason for so many functions of lncRNAs in the cells is that lncRNAs can crosstalk with miRNAs as a competing endogenous RNA (ceRNA). ${ }^{36}$ Therefore, we searched for the potential targets of SNHG17 in CC and found that miR-375-3p may be a direct target of SNHG17 in CC. This correlation mentioned oral squamous cell carcinoma by Tong's too. ${ }^{14}$ In this study, we found miR-375-3p expression was inversely correlated with SNHG17 expression in CC patients. Subsequently, it is validated that miR$375-3 p$ was negatively regulated by SNHG17. Luciferase reporter assay demonstrated that miR-375$3 p$ mimics or inhibitors could obviously influence OD values of WT-SNHG17 plasmid and implied miR-375$3 p$ bind to the 3 'UTR of SNHG17. Furthermore, rescue experiments suggested that knockdown of SNHG17 inhibited cell growth through repressing miR-375-3p expression at least in part. Succinctly, the results verified that SNHG17- miR-375-3p axis might be the key point of SNHG17 function in CC. On the basis of the researches along with the current study, we reckon that
SNHG17 suppresses CC cell proliferation, migration and invasion by targeting miR-375-3p.

The results of this study are promising in a certain extent. However, some limitations also exist: (i) limited to the number of $\mathrm{CC}$ cases in our single hospital, further studies getting a larger sample size involved in are recommended; (ii) it is uncertain whether alteration of SNHG17 and miR-375-3p expression in the serum is particular for $\mathrm{CC}$ or its certain sub-types, which need additional researches challenging against other cancers or various $\mathrm{CC}$ subtypes; (iii) the confirmation of expression level of SNHG17 and miR-375-3p in the CC resected biopsy is needed in the future.

\section{Conclusion}

In conclusion, the results in this study indicate that the overexpression of SNHG17 can promote the progression of CC via suppressing the expression of miR-3753p. SNHG17 and miR-375-3p have obvious clinical significance in $\mathrm{CC}$ and can act as candidate indicators of diagnosis and potential therapeutic targets for CC. 
A

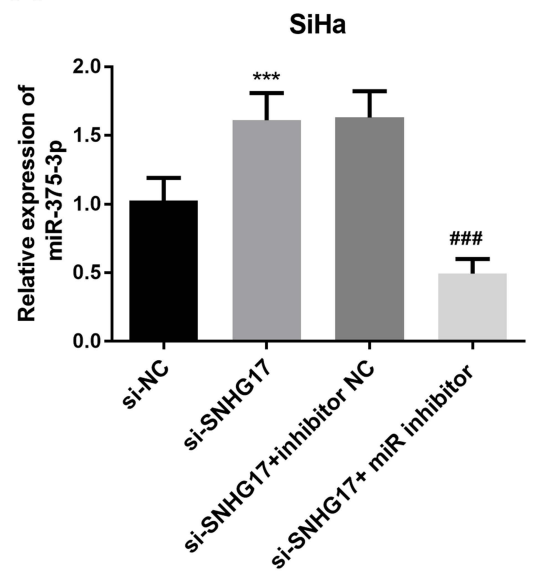

D

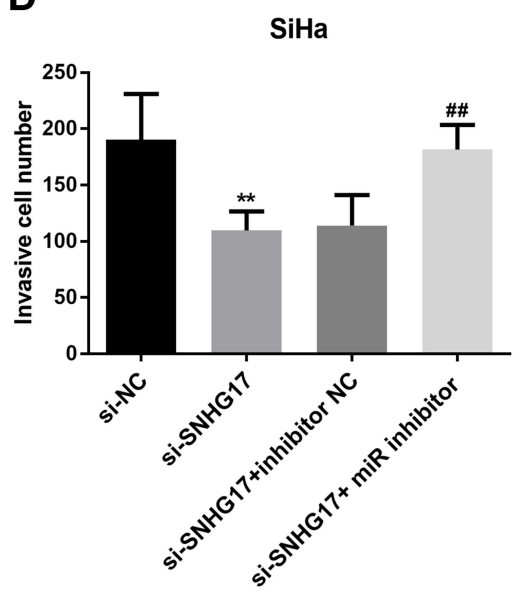

B

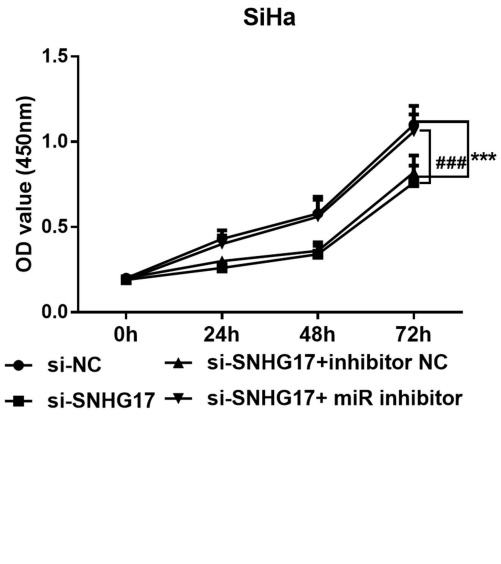

C

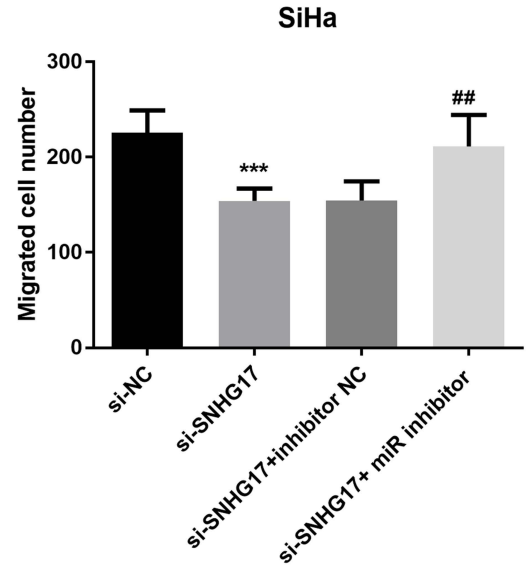

E

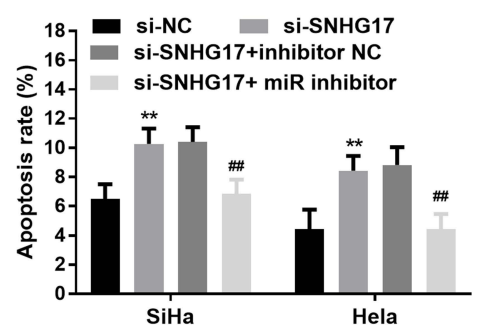

Figure 5 Rescue experiments verified the negative correlation between SNHGI7 and miR-375-3p. (A) Reducing the expression of SNHGI7 caused an increased expression of miR-375-3p, but miR-375-3p inhibitor can bring down this increase. (B) Downregulated SNHGI7 inhibits CC cell proliferation, but miR-375-3p inhibitor abolished the suppressive effect. (C) CC cell migration was inhibited by knockdowning SNHGI7 expression, but rescued by miR-375-3p inhibitor. (D) CC cell invasion was repressed by knockingdowning SNHGI7 expression, but recuperated by miR-375-3p inhibitor. (E) miR-375-3p inhibitor can prevent the CC cell apoptosis induced by Downregulated SNHG I7. ${ }^{* *} p<0.01$, ${ }^{* * *} p<0.001$ (compared with si-NC group); ${ }^{\#} p<0.01$, ${ }^{\# \#} p<0.001$ (compared with si- SNHGI7 + miR-375-3p inhibitor group).

\section{Ethics Statement}

The protocol of this study was approved by the institutional ethics review board of Dongying District People's Hospital. Signed written consent was acquired by the patients and healthy volunteers before the initiation of any study-related procedure. Patients and healthy volunteers were informed about the purpose of the study, and that it was conducted in accordance with the Declaration of Helsinki.

\section{Disclosure}

The authors report no conflicts of interest in this work.

\section{References}

1. Fowler JR, Jack BW. Cervical Cancer. StatPearls. StatPearls Publishing. Copyright (C) 2020, StatPearls Publishing LLC; 2020.
2. Cohen PA, Jhingran A, Oaknin A, Denny L. Cervical cancer. Lancet. 2019;393(10167):169-182. doi:10.1016/S0140-6736(18)32470-X

3. Arbyn M, Weiderpass E, Bruni L, et al. Estimates of incidence and mortality of cervical cancer in 2018: a worldwide analysis. Lancet Glob Health. 2020;8(2):e191-e203. doi:10.1016/S2214-109X(19) 30482-6

4. Salunkhe R, Chopra S, Kulkarni S, et al. Outcomes of locally advanced cervical cancer presenting with obstructive uropathy: an institutional audit. Indian $J$ Cancer. 2020;57(4):416-422. doi:10.4103/ijc.IJC 70418

5. Tsikouras P, Zervoudis S, Manav B, et al. Cervical cancer: screening, diagnosis and staging. $J$ buon. 2016;21(2):320-325.

6. Ngo-Metzger Q, Adsul P. Screening for cervical cancer. Am Fam Physician. 2019;99(4):253-254.

7. Goodall GJ, Wickramasinghe VO. RNA in cancer. Nat Rev Cancer. 2021;21(1):22-36. doi:10.1038/s41568-020-00306-0

8. Yarmishyn AA, Kurochkin IV. Long noncoding RNAs: a potential novel class of cancer biomarkers. Front Genet. 2015;6:145. doi:10.3389/fgene.2015.00145

9. Yang B, Zhang L, Cao Y, et al. Overexpression of lncRNA IGFBP41 reprograms energy metabolism to promote lung cancer progression. Mol Cancer. 2017;16(1):154. doi:10.1186/s12943-017-0722-8 
10. Shima H, Kida K, Adachi S, et al. Lnc RNA H19 is associated with poor prognosis in breast cancer patients and promotes cancer stemness. Breast Cancer Res Treat. 2018;170(3):507-516. doi:10.1007/s10549-018-4793-Z

11. Li Q-G, Xu X-Q, Zhou D-Y, et al. Long non-coding RNA DILC as a potentially useful biomarker for the diagnosis and prognosis of colorectal cancer. Eur Rev Med Pharmacol Sci. 2019;23 (8):3320-3325. doi:10.26355/eurrev_201904_17694

12. Mahmoudian-Sani M-R, Jalali A, Jamshidi M, et al. Long non-coding RNAs in thyroid cancer: implications for pathogenesis, diagnosis, and therapy. Oncol Res Treat. 2019;42(3):136-142. doi:10.1159/ 000495151

13. Pan X, Guo Z, Chen Y, et al. STAT3-induced lncRNA SNHG17 exerts oncogenic effects on ovarian cancer through regulating CDK6. Mol Ther Nucleic Acids. 2020;22:38-49. doi:10.1016/j.omtn.2020.08.006

14. Tong F, Guo J, Miao Z, Li Z. LncRNA SNHG17 promotes the progression of oral squamous cell carcinoma by modulating miR-375/PAX6 axis. Cancer Biomark. 2021;30(1):1-12. doi:10.3233/CBM-191070

15. Li W, Zheng Y, Mao B, Wang F, Zhong Y, Cheng D. SNHG17 upregulates WLS expression to accelerate lung adenocarcinoma progression by sponging miR-485-5p. Biochem Biophys Res Commun. 2020;533(4):1435-1441. doi:10.1016/j.bbrc.2020.09.130

16. Xu T, Yan S, Jiang L, et al. Gene amplification-driven long noncoding RNA SNHG17 regulates cell proliferation and migration in human non-small-cell lung cancer. Mol Ther Nucleic Acids. 2019;17:405-413. doi:10.1016/j.omtn.2019.06.008

17. Du F, Hou Q. SNHG17 drives malignant behaviors in astrocytoma by targeting miR-876-5p/ERLIN2 axis. BMC Cancer. 2020;20(1):839. doi:10.1186/s12885-020-07280-8

18. Liu J, Zhan Y, Wang J, Wang J, Guo J, Kong D. IncRNA-SNHG17 promotes colon adenocarcinoma progression and serves as a sponge for miR-375 to regulate CBX3 expression. Am J Transl Res. 2020;12 (9):5283-5295.

19. Babion I, Snoek BC, Novianti PW, et al. Triage of high-risk HPV-positive women in population-based screening by miRNA expression analysis in cervical scrapes; a feasibility study. Clin Epigenetics. 2018;10(1):76. doi:10.1186/s13148-018-0509-9

20. Ma Z, Gu S, Song M, et al. Long non-coding RNA SNHG17 is an unfavourable prognostic factor and promotes cell proliferation by epigenetically silencing P57 in colorectal cancer. Mol Biosyst. 2017;13(11):2350-2361. doi:10.1039/C7MB00280G

21. Wu Y, Sun X, Song B, Qiu X, Zhao J. MiR-375/SLC7A11 axis regulates oral squamous cell carcinoma proliferation and invasion. Cancer Med. 2017;6(7):1686-1697. doi:10.1002/cam4.1110

22. Jules J, Maiguel D, Hudson BI, Oury TD. Alternative splicing of the RAGE cytoplasmic domain regulates cell signaling and function. PLoS One. 2013;8(11):e78267. doi:10.1371/journal.pone.0078267

23. van de Merbel AF, van der Horst G, Buijs JT, van der Pluijm G. Protocols for migration and invasion studies in prostate cancer. Methods Mol Biol. 1786;67-79.
24. McGuire A, Brown JAL, Kerin MJ. Metastatic breast cancer: the potential of miRNA for diagnosis and treatment monitoring. Cancer Metastasis Rev. 2015;34(1):145-155. doi:10.1007/s10555-015-9551-7

25. Solé C, Moliné T, Vidal M, Ordi-Ros J, Cortés-Hernández J. An exosomal urinary miRNA signature for early diagnosis of renal fibrosis in lupus nephritis. Cells. 2019;8(8). doi:10.3390/ cells 8080773

26. Tutar Y. Editorial (Thematic issue: "miRNA and cancer; computational and experimental approaches"). Curr Pharm Biotechnol. 2014;15(5):429. doi:10.2174/138920101505140828161335

27. Mishra PK, Nemer G. Editorial: the non-coding genome and cardiovascular disease. Front Cardiovasc Med. 2019;6:98. doi:10.3389/ fcvm.2019.00098

28. Yao Z, Jia C, Tai Y, et al. Serum exosomal long noncoding RNAs lnc-FAM72D-3 and lnc-EPC1-4 as diagnostic biomarkers for hepatocellular carcinoma. Aging. 2020;12(12):11843-11863. doi:10.18632/aging.103355

29. Liu L, Shuai T, Li B, Zhu L, Li X. Long non-coding RNA lncGNAT1-1 inhibits gastric cancer cell proliferation and invasion through the Wnt/ $\beta$-catenin pathway in Helicobacter pylori infection. Mol Med Rep. 2018;18(4):4009-4015. doi:10.3892/mmr.2018.9405

30. Chen -L-L, He J, Qiu X-T, Yu J, Wang Z-M. The prognostic roles of long non-coding RNA SNHG17 in the patients with gastric cancer. Eur Rev Med Pharmacol Sci. 2019;23(3):1063-1068. doi:10.26355/ eurrev_201902_16994

31. Liu Y, Li Q, Tang D, et al. SNHG17 promotes the proliferation and migration of colorectal adenocarcinoma cells by modulating CXCL12-mediated angiogenesis. Cancer Cell Int. 2020;20(1):566. doi:10.1186/s12935-020-01621-0

32. Wu G, Hao C, Qi X, et al. LncRNA SNHG17 aggravated prostate cancer progression through regulating its homolog SNORA71B via a positive feedback loop. Cell Death Dis. 2020;11(5):393. doi:10.1038/s41419-020-2569-y

33. Du Y, Wei N, Hong J, Pan W. Long non-coding RNASNHG17 promotes the progression of breast cancer by sponging miR-124-3p. Cancer Cell Int. 2020;20(1):40. doi:10.1186/s12935-020-1129-y

34. Zheng Z-J, Liu Y, Wang H-J, Pang -W-W, Wang Y. LncRNA SNHG17 promotes proliferation and invasion of ovarian cancer cells through up-regulating FOXA1. Eur Rev Med Pharmacol Sci. 2020;24(18):9282-9289. doi:10.26355/eurrev 20200923010

35. Yu X, Zhao W, Yang X, Wang Z, Hao M. miR-375 affects the proliferation, invasion, and apoptosis of HPV16-positive human cervical cancer cells by targeting IGF-1R. Int $J$ Gynecol Cancer. 2016;26(5):851-858. doi:10.1097/IGC.0000000000000711

36. Kim J, Abdelmohsen K, Yang X, et al. LncRNA OIP5-AS1/cyrano sponges RNA-binding protein HuR. Nucleic Acids Res. 2016;44 (5):2378-2392. doi:10.1093/nar/gkw017

\section{Publish your work in this journal}

Cancer Management and Research is an international, peer-reviewed open access journal focusing on cancer research and the optimal use of preventative and integrated treatment interventions to achieve improved outcomes, enhanced survival and quality of life for the cancer patient.
The manuscript management system is completely online and includes a very quick and fair peer-review system, which is all easy to use. Visit http://www.dovepress.com/testimonials.php to read real quotes from published authors. 